\title{
Computational prediction and analysis of impact of the cross-talks between JNK and P38 kinase cascades
}

\author{
Pandurangan Sundaramurthy ${ }^{1,2}$, Sunita Gakkhar ${ }^{1,2}$ and Ramanathan Sowdhamini ${ }^{2,}$ * \\ ${ }^{1}$ Department of Mathematics, Indian Institute of Technology Roorkee, Roorkee - 247667, Uttarakhand, India; ${ }^{2}$ National Center for \\ Biological Sciences, Tata Institute of Fundamental Research, UAS-GKVK campus, Bellary Road, Bangalore - 560 065, India; \\ R. Sowdhamini* - Email: mini@ncbs.res.in ;* Corresponding author
}

received November 04, 2008; accepted November 30, 2008; published January 12, 2009

\begin{abstract}
:
Signal transduction is a complex protein signaling process with a rich network of multifunctional interactions that occur in a non-linear fashion. Mitogen-activated protein kinase (MAPK) signal transduction pathways regulate diverse cellular processes ranging from proliferation and differentiation to apoptosis. In mammals, out of five, there are three well characterized subfamilies of MAPKs - ERKs (Extracellular signal-regulated kinases), JNKs (c-Jun N-terminal kinases), and P38 kinases, and their activators, are implicated in human diseases and are targets for drug development. Kinase cascades in MAPK pathways mediate the sensing and processing of stimuli. To understand how cells makes decisions, the dynamic interactions of components of signaling cascades are important rather than just creating static maps. Based on enzyme kinetic reactions, we have developed a mathematical model to analyze the impact of the cross-talks between JNK and P38 kinase cascades. Cross-talks between JNK and P38 kinase cascades influence the activities of P38 kinases. Responses of the signals should be studied for network of kinase cascades by considering cross-talks.
\end{abstract}

Keywords: cross-talks; dynamic pathway modeling; JNK and P38 kinase cascades; signaling pathways

\section{Background:}

All cells receive and respond to signals from their environment, whether they live freely or are part of a tissue. Network of signaling pathways detect, amplify, and integrate diverse external signals to generate responses such as changes in enzyme activity, gene expression, or ion-channel activity to regulate virtually all aspects of cell behavior, including metabolism, movement, proliferation, survival, and differentiation.

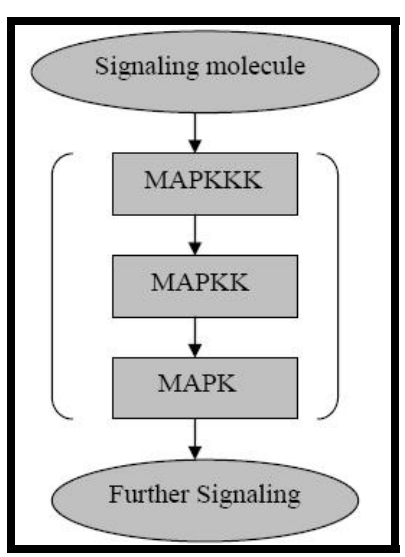

Figure 1: MAPK cascade

Mitogen-activated protein kinases (MAPKs) compose a family of protein kinases whose function and regulation have been conserved during evolution from unicellular organisms such as brewers' yeast to complex organisms including humans, connecting cell-surface receptors to

ISSN 0973-2063 (online) 0973-8894 (print)

Bioinformation 3(6): 250-254 (2009) critical regulatory targets within cells. MAPK activity is regulated through three-tiered cascades composed of a MAPK, MKK or MAPKK (Mitogen-activated protein kinase kinase) and MEKK or MAPKKK (Mitogenactivated protein kinase kinase kinase) (Figure 1) [1]. In mammals, out of five, there are three well characterized subfamilies of MAPKs - ERKs (Extracellular signalregulated kinases), JNKs (c-Jun N-terminal kinases), and P38 kinases [2]. Based on the Information's given in JNK and P38 pathway diagrams deposited in the Science's STKE(Signal Transduction Knowledge Environment) database [3, 4], we have drawn the diagram (Figure 2) to depict the JNK and P38 Kinase cascades with cross- talks.

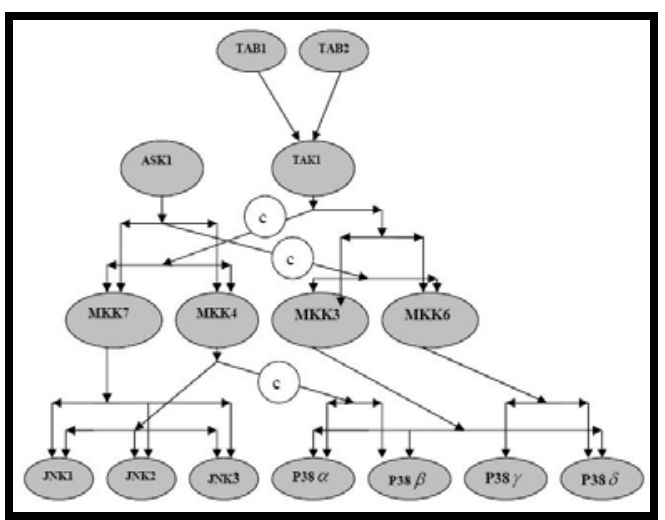

Figure 2: JNK and P38 kinase cascades with cross-talks. C $=$ cross talks 
The c-Jun N-terminal Kinases consist of three isoforms. JNK1 and JNK2 are the products of alternative splicing of a single gene and are expressed in many tissues, but JNK3 is specifically expressed in neuronal tissue brain. Members of the JNK family play crucial roles in regulating responses to environmental stress, radiation, and growth factors, and in neural development, inflammation, and apoptosis [5]. Four isoforms of P38 MAP kinase, P38 $\alpha$, $\mathrm{P} 38 \beta$, P38 $\gamma$ and P38 $\delta$ have been identified. The P38 MAPKs play an important role in asthma and autoimmunity in humans and are activated by numerous physical and chemical stresses, including hormones, UV irradiation, ischemia, cytokines including interleukin-1 and tumor necrosis factor, osmotic shock and heat shock [6].

In the processes of cellular signaling, protein-protein interactions play a central role. Protein kinases are enzymes that covalently attach phosphate to the side chain of serine, threonine, or tyrosine of specific proteins inside cells and protein phosphatases remove the phosphates that were transferred to the protein substrate by the kinase. In this manner, the action of MAPKs and protein phosphatases reciprocally and rapidly alter the behavior of cells as they respond to changes in their environment [6]. A MAPKKK that is activated by extra cellular stimuli phosphorylates a MAPKK on its serine and threonine residues, and then this MAPKK activates a MAPK through phosphorylation on its threonine and tyrosine residues and then this MAPKs phosphorylate specific serines of target protein substrates and regulate cellular activities ranging from gene expression, mitosis, movement, metabolism, and programmed cell death [6].

\section{Methodology:}

Systems approach

Cataloguing and classification of signaling molecules will ultimately not suffice to reason out the function of signaling networks or functioning of cells [7], but by the integration of this information through mathematical modeling and subsequent simulation of "networks" of "pathways" composed of interacting (macro-) molecules. We depicts a set of structural relationships among its components of JNK and P38 Kinase cascades (Figure 2) and therefore, demands to be converted into a set of mathematical equations that describe the temporal and spatial evolution of the system.

\section{Dynamic pathway modeling}

Differential equation models are well defined encodings of molecular interactions contributing towards the synthesis and degradation of a protein in the context of cell signaling. A basic assumption of this approach is that the cell presents a well-stirred biochemical reactor. It is known that, as the signal transfer between the cell surface and the nucleus occurs in a stepwise manner, a systems biologist will also think of a step by step biochemical reaction of the whole pathway with network systems of interacting signaling substances receiving inputs and engendering outputs [8].

See supplementary material for mathematical formulation. References [9], [10] and [11] are used in supplementary material.

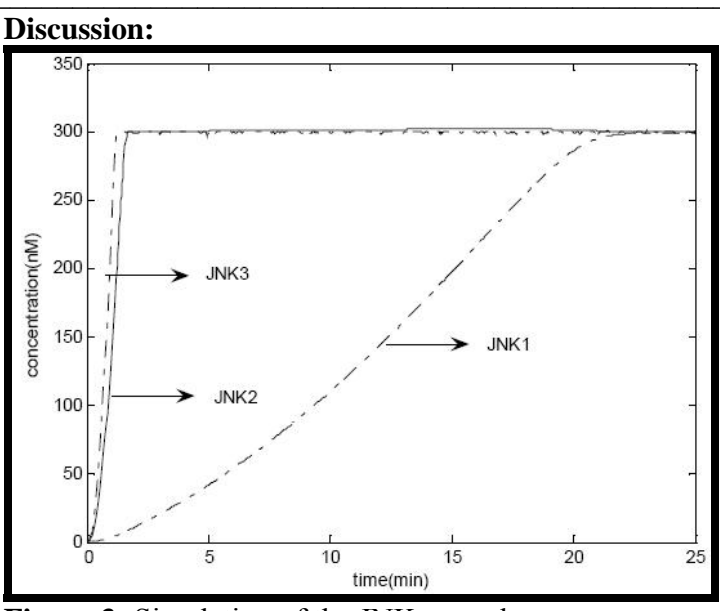

Figure 3: Simulation of the JNK cascade

We have used Adams-Bashforth numerical algorithm and MATLAB programming to simulate the system of differential equations. Availability of quantitative values for molar concentrations and reaction rate constants has been a bottleneck for the researchers who are interested to study the dynamic behavior of the signaling pathways for which pathway diagram alone deposited in the databases. Since the molar concentrations and reaction rate constants are not same in the cell types and organisms, we have assumed reasonable values for the parameters representing them in concurrence with the values used in the other MAPK pathway modeling works published in the journals. In this work, for the sack of analysis, we have considered only the plots which represent molar concentrations of the activated JNK and P38 kinases. The plots in (Figure 3) are obtained by simulating the system of equations (3a-3e under supplementary material) without cross-talk terms, which represents the dynamic model of JNK cascade. The plots in (Figure 4) are obtained by simulating the system of equations (3f-3l, see supplementary material) without cross-talk terms, which represents the dynamic model of P38 kinase cascade responses to unit-step input signals. The plots in (Figure 5) are obtained by simulating the system of equations (3a-31, under supplementary material), which represents the dynamic model of JNK and P38 Kinase cascades with cross-talks, responses to unit-step input signals.

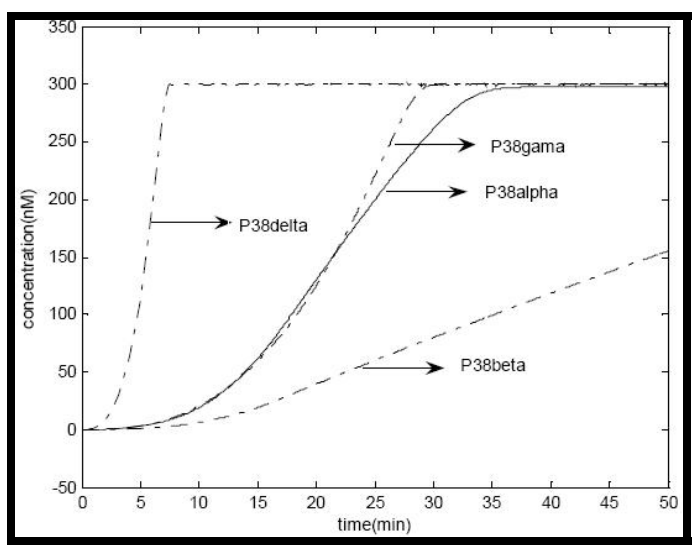

Figure 4: Simulation of the P38 kinase cascade with unitstep input signal. 
We have observed that, the system representing the JNK and P38 Kinase cascades with cross - talks is robust in nature. For the range of input signals $(1 \mathrm{nM}-5 \mathrm{nM})$, the range of reaction rate constants $(0.001 \mathrm{nM} / \mathrm{s}-3 \mathrm{nM} / \mathrm{s})$, and the range of molar concentrations of kinases $(100 \mathrm{nM}-$ $300 \mathrm{nM}$ ), the ultra sensitive / sustained / transient manner JNK and P38 Kinases activation doest not vary, only the time taken by the activated JNK's and P38's to reach the saturation is varying. Also, we have observed that, ultra sensitive and sustained manner of JNK2 and JNK3 activation and the transient manner of JNK1 activation does not get affected due to cross - talks. But, it is due to cross - talks, P38 $\alpha$ kinases become inactivated, transiently activated P38 $\beta$ kinases reaches saturation earlier, and transiently activated P38 $\gamma$ kinases are activated in ultra sensitive and sustainable manner.

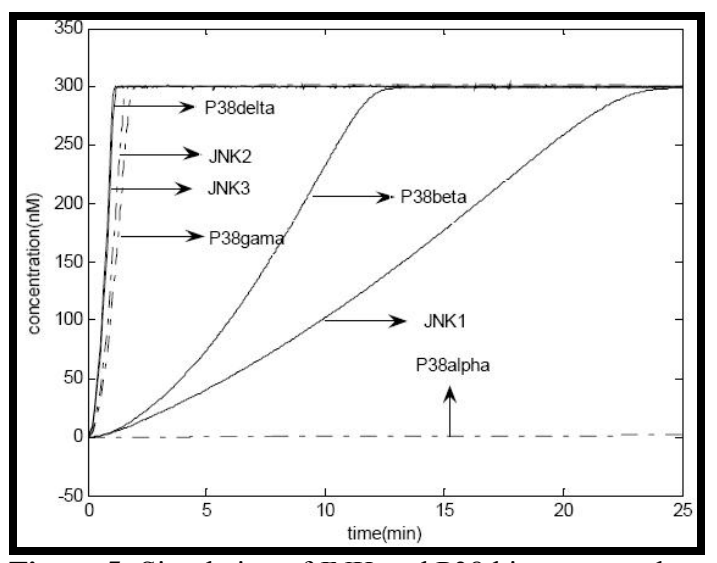

Figure 5: Simulation of JNK and P38 kinase cascades with cross-talks and unit-step input signal.

In general, the sustained JNK2 and JNK3 activation might associate with apoptosis and the transient JNK1 activation might associate with survival [12]. Specifically, the sustained JNK2 and JNK3 activation in fibroblasts may result in a pro-apoptotic function and the transient JNK1 activation may result in proliferation [13]. The sustained P38 $\gamma$ kinases activation due to the cross - talks might mediate process like mitogenesis, cell fate induction [14], and may result in an anti-angiogenic phenotype that contributes to endothelial dysfunction [15]. Also, we have observed that, it is due to cross talks, the P38 $\alpha$ kinases lost its functional role by not getting activated and P38 $\beta$ kinases activation become more transient.

\section{Conclusion:}

Cross-talks between JNK and P38 kinase cascades influence the activities of P38 kinases. Responses of the signals should be studied for network of kinase cascades by considering cross-talks.

\section{References:}

[01] C. Widmann et al., Physiol Rev., 79: 143 (1999) [PMID: 9922370]

[02] J. M. English and M.H. Cobb English, Trends Pharmacol Sci., 23: 40 (2002) [PMID: 11804650].

[03] http://stke.sciencemag.org/cgi/cm/stkecm;CMP_10 827

[04] http://stke.sciencemag.org/cgi/cm/stkecm;CMP_10 958

[05] C. Tournier et al., Science, 288: 870 (2000) [PMID: 10797012]

[06] G. L. Johnson and R. Lapadat, Science, 298: 1911 (2002) [PMID: 12471242]

[07] L. Chang and M. Karin, Nature, 410: 37 (2001) [PMID: 11242034]

[08] C. V. Suresh Babu et al., Biochimie, 88: 277 (2006) [PMID: 16213652]

[09] O. Wolkenhauer et al., Biochem SocTrans., 33: 507 (2005) [PMID: 15916553]

[10] R. Heinrich et al., Mol Cel., 9: 957(2002) [PMID: 12049733]

[11] B. N. Kholodenko et al., Proc Natl Acad Sci U S A., 99: 12841(2002) [PMID: 12242336]

[12] C. Reinhard et al., EMBO J., 16: 5 (1997) [PMID: 9118946]

[13] E. Shaulian et al., Cell, 103: 6 (2000) [PMID: 11136975]

[14] J. E. Ferrell Jr., Trends Biochem Sci., 21: 12(1996) [PMID: 9009826]

[15] M. E. McMullen et al., J Biol Chem., 280: 22 (2005)[ PMID: 15790570]

Citation: Sundaramurthy et al., Bioinformation 3(6): 250-254 (2009)

License statement: This is an open-access article, which permits unrestricted use, distribution, and reproduction in any medium, for non-commercial purposes, provided the original author and source are credited.

\section{Supplementary material}

Methodology:

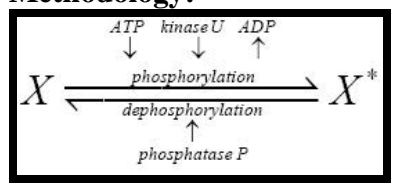

Figure S1: Biochemical reaction

In signaling, activation/inactivation of proteins corresponds to phosphorylation/ dephosphorylation [9]. Denoting the kinase as $U$, the phosphatase as $P$ and the protein as $X$, and assuming a constant phosphatase, (Figure S1) illustrates a simple

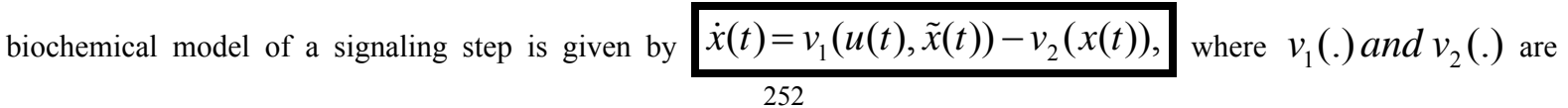




\section{www.bioinformation.net}

Hypothesis

mappings, describing the reaction rates for phophorylation and dephophorylation respectively [9]. We write $\tilde{X}(t)$ for the non - phosphorylated form of the protein $X, u(t)$ for the kinase $U$, and $x(t)$ correspond to the activated protein $X^{*}$. Referring to a power-law representation, one would have $v_{1}(u(t), \tilde{x}(t))=k_{1} u(t)^{a} \tilde{x}(t)^{b}, v_{2}(x(t))=k_{2} x(t)^{c}$ where we choose $a=b=c=1$, such that $\dot{x}=k_{1} u(t) \tilde{x}(t)-k_{2} x(t)$. If we assume that the total $\bar{x}=\tilde{x}(t)+x(t)$ is constant for all time $t$, we require only this one differential equation $\dot{x}=k_{1} u(t)(\bar{x}(t)-x(t))-k_{2} x(t)$ to model a signaling step [10]. In our model we have assumed Michaelis-Menten kinetics [11].

\begin{tabular}{|c|c|}
\hline$=\frac{k_{1} u(t)(\bar{x}(t)-x(t))}{2}$ & $k_{2} x(t)$ \\
\hline$K m 1+(\bar{x}(t)-x(t))$ & $K m 2+x(t)$ \\
\hline & \\
\hline
\end{tabular}

Modeling JNK and P38 kinase cascades with cross-talks

To model the entire JNK and P38 Kinase cascades with cross-talks (Figure 2), we have the following state variables representing the concentration of each protein involved in the system (Figure 2). Let $u=$ ASK1, $v_{1}=$ TAB1 (TAK1 (Transforming growth factor-beta-activated kinase 1)binding protein 1), $v_{2}=$ TAB2 (TAK1 binding protein 2), $x_{1}(t)=\mathrm{MKK} 7, \quad x_{2}(t)=\mathrm{MKK} 4, \quad x_{3}(t)=\mathrm{JNK} 1, \quad x_{4}(t)=\mathrm{JNK} 2, \quad x_{5}(t)=\mathrm{JNK} 3, \quad y_{1}(t)=\mathrm{TAK} 1, \quad y_{2}(t)=\mathrm{MKK} 3$, $y_{3}(t)=\mathrm{MKK} 6, \quad y_{4}(t)=\mathrm{P} 38 \alpha, \quad y_{5}(t)=\mathrm{P} 38 \beta, \quad y_{6}(t)=\mathrm{P} 38 \gamma, \quad$ and $\quad y_{7}(t)=\mathrm{P} 38 \delta . \quad$ Also, let $\left\{x_{i}(t)\right\}_{i=1 \text { to5 }}$ and $\left\{y_{j}(t)\right\}_{j=1 \text { to } 7}$ are the activated (phosphorylated) form of the proteins and the tilted ones are corresponds to the inactivated (non-phosphorylated) in JNK and P38 Kinase cascades respectively. We assume that the total $\left\{\bar{x}_{i}(t)=\tilde{x}_{i}(t)+x_{i}(t)\right\}_{i=1 t o 5}$ and $\left\{\bar{y}_{j}(t)=\tilde{y}_{j}(t)+y_{j}(t)\right\}_{j=1 t o 7}$ is constant for all $\mathrm{t}$.

\begin{tabular}{|c|c|c|}
\hline$c_{1} \underset{\begin{array}{c}u \\
\downarrow\end{array}}{\stackrel{\substack{\downarrow \\
k_{1}, c_{1}, c_{1}^{\prime}}}{\rightleftharpoons}} x_{1}$ & $\tilde{x}_{2} \underset{\begin{array}{ccc}u & y_{1} \\
& \downarrow & \downarrow \\
k_{2}, & c_{2}\end{array}}{\stackrel{k_{2}^{\prime}, c_{3}^{\prime}}{\rightleftharpoons}} x_{2}$ & 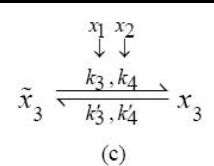 \\
\hline$\tilde{x}_{4} \frac{\begin{array}{cc}x_{1} & x_{2} \\
\downarrow & \downarrow \\
k_{5}, k_{6}\end{array}}{\underset{\begin{array}{l}k_{5}^{\prime}, k_{6}^{\prime} \\
\text { (d) }\end{array}}{\text { (d) }}} x_{4}$ & 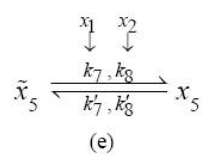 & 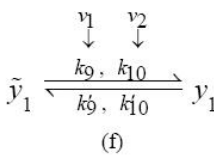 \\
\hline 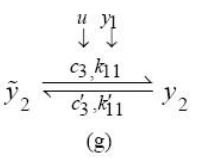 & 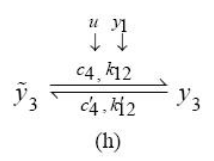 & $\tilde{y}_{4} \stackrel{\begin{array}{c}x_{2} y_{2} \\
\downarrow \\
\downarrow \\
c_{5}, k_{13}\end{array}}{\underset{c_{5}^{\prime}, k_{13}^{\prime}}{\text { (i) }}} y_{4}$ \\
\hline 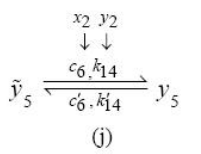 & 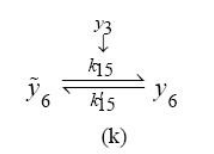 & 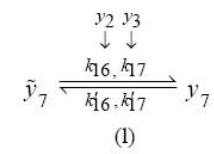 \\
\hline
\end{tabular}

Figure S2: (a) to (1): Reaction schemes involved in activation (phosphorylation) and inactivation (de-phosphorylation) of all the proteins in JNK and P38 kinase cascades with cross-talks

Based on the reaction schemes described in (FigureS2 (a) - 4(1)), a set of differential equations (3a)- (31) have been developed to form the dynamic system, to analyze the impact of cross talks between the JNK and P38 Kinase cascades (Figure 2).

$\frac{d x_{1}(t)}{d t}=\frac{k_{1} u\left(\bar{x}_{1}-x_{1}(t)\right)}{K m_{1}+\left(\bar{x}_{1}-x_{1}(t)\right)}+\frac{c_{1} y_{1}(t)\left(\bar{x}_{1}-x_{1}(t)\right)}{C m_{1}+\left(\bar{x}_{1}-x_{1}(t)\right)}-\frac{k_{1}^{\prime} x_{1}(t)}{K^{\prime} m_{1}+x_{1}(t)}-\frac{c_{1}^{\prime} x_{1}(t)}{C^{\prime} m_{1}+x_{1}(t)}$




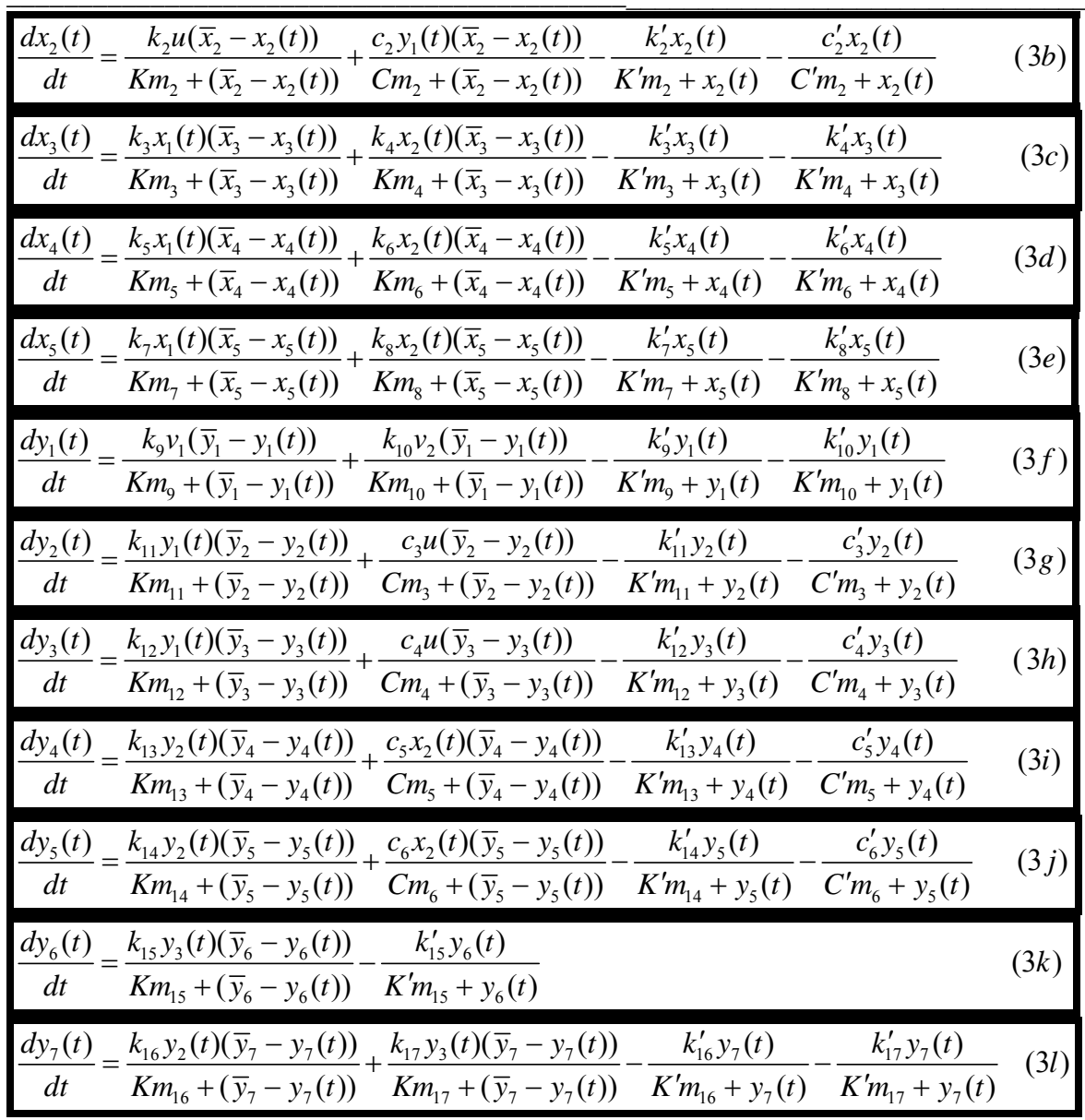

Where $k_{i}, k_{i}^{\prime} ; i=1$ to 17 and $c_{j}, c_{j}^{\prime} ; j=1$ to 6 are reactions rate constants and

$\mathrm{Km}_{i}, K^{\prime} m_{i} ; i=1$ tol 7 and $\mathrm{Cm}_{j}, \mathrm{C}^{\prime} \mathrm{m}_{j} ; j=1$ to6 are Michaelis-Menten's constants. The parameter values are given in Table 1 (supplementary materail).

Eq. no. $\quad$ Parameter values

(3a) $\mathrm{k}_{1}=2.5, \mathrm{k}_{1}^{\prime}=0.025, \mathrm{Km}_{1}=15, \mathrm{~K}^{\prime} \mathrm{m}_{1}=15, \mathrm{c}_{1}=1.05, \mathrm{c}_{1}^{\prime}=0.05, \mathrm{Cm}_{1}=15$, $\mathrm{C}^{\prime} \mathrm{m}_{1}=15, \mathrm{u}=100, \overline{\mathrm{x}}_{1}=300$

(3b) $\mathrm{k}_{2}=0.025, \mathrm{k}_{2}^{\prime}=0.75, \mathrm{Km}_{2}=15, \mathrm{~K}^{\prime} \mathrm{m}_{2}=15, \mathrm{c}_{2}=0.03, \mathrm{c}_{2}^{\prime}=2.05, \mathrm{Cm}_{2}=15$, $\mathrm{C}^{\prime} \mathrm{m}_{2}=15, \overline{\mathrm{x}}_{2}=310$

(3c) $\mathrm{k}_{3}=1.25, \mathrm{k}_{3}^{\prime}=0.5, \mathrm{Km}_{3}=15, \mathrm{~K}^{\prime} \mathrm{m}_{3}=15, \mathrm{k}_{4}=0.5, \mathrm{k}_{4}^{\prime}=1.5, \mathrm{Km}_{4}=15$, $\mathrm{K}^{\prime} \mathrm{m}_{4}=15, \overline{\mathrm{x}}_{3}=300$,

(3d) $\mathrm{k}_{5}=1.25, \mathrm{k}_{5}^{\prime}=0.015, \mathrm{Km}_{5}=15, \mathrm{~K}^{\prime} \mathrm{m}_{5}=15, \mathrm{k}_{6}=2.25, \mathrm{k}_{6}^{\prime}=0.019, \mathrm{Km}_{6}=15$, $\mathrm{K}^{\prime} \mathrm{m}_{6}=15, \overline{\mathrm{x}}_{4}=300$,

(3e) $\mathrm{k}_{7}=0.25, \mathrm{k}_{7}^{\prime}=1.15, \mathrm{Km}_{7}=15, \mathrm{~K}^{\prime} \mathrm{m}_{7}=15, \mathrm{k}_{8}=0.025, \mathrm{k}_{8}^{\prime}=1.05$, $\mathrm{Km}_{8}=15, \mathrm{~K}^{\prime} \mathrm{m}_{8}=15, \overline{\mathrm{x}}_{5}=300$,

(3f) $\mathrm{k}_{9}=2.5, \mathrm{k}_{9}^{\prime}=0.05, \mathrm{Km}_{9}=10, \mathrm{~K}^{\prime} \mathrm{m}_{9}=8, \mathrm{k}_{10}=0.05, \mathrm{k}_{10}^{\prime}=1.5, \mathrm{Km}_{10}=10$, $\mathrm{K}^{\prime} \mathrm{m}_{10}=8, \mathrm{v}_{1}=1, \mathrm{v}_{2}=1, \overline{\mathrm{y}}_{1}=100$

(3g) $\mathrm{k}_{11}=2.6, \mathrm{k}_{11}^{\prime}=0.075, \mathrm{Km}_{11}=15, \mathrm{~K}^{\prime} \mathrm{m}_{11}=15, \mathrm{c}_{3}=0.06, \mathrm{c}_{3}^{\prime}=2.6, \mathrm{Cm}_{3}=15$, $\mathrm{C}^{\prime} \mathrm{m}_{3}=15, \overline{\mathrm{y}}_{2}=300$ (3h) $\mathrm{k}_{12}=0.02, \mathrm{k}_{12}^{\prime}=2.01, \mathrm{Km}_{12}=15, \mathrm{~K}^{\prime} \mathrm{m}_{12}=15, \mathrm{c}_{4}=2.5, \mathrm{c}_{4}^{\prime}=1.5, \mathrm{Cm}_{4}=15$, $\mathrm{C}^{\prime} \mathrm{m}_{4}=15, \overline{\mathrm{y}}_{3}=300$

(3i)

$\mathrm{k}_{13}=1.03, \mathrm{k}_{13}^{\prime}=0.09, \mathrm{Km}_{13}=15, \mathrm{~K}^{\prime} \mathrm{m}_{13}=15, \mathrm{c}_{5}=0.05, \mathrm{c}_{5}^{\prime}=1.5, \mathrm{Cm}_{5}=15$, $\mathrm{C}^{\prime} \mathrm{m}_{5}=15, \overline{\mathrm{y}}_{4}=300$

(3j)

$\mathrm{k}_{14}=2.03, \mathrm{k}_{14}^{\prime}=0.05, \mathrm{Km}_{14}=15, \mathrm{~K}^{\prime} \mathrm{m}_{14}=15, \mathrm{c}_{6}=2.5, \mathrm{c}_{6}^{\prime}=1.05, \mathrm{Cm}_{6}=15$, $\mathrm{C}^{\prime} \mathrm{m}_{6}=15, \overline{\mathrm{y}}_{5}=300$

(3k) $\mathrm{k}_{15}=1.03, \mathrm{k}_{15}^{\prime}=0.03, \mathrm{Km}_{15}=15, \mathrm{~K}^{\prime} \mathrm{m}_{15}=15, \overline{\mathrm{y}}_{6}=300$

(3l) $\mathrm{k}_{16}=2.03, \mathrm{k}_{16}^{\prime}=1.03, \mathrm{Km}_{16}=15, \mathrm{~K}^{\prime} \mathrm{m}_{16}=15, \mathrm{k}_{17}=1.03, \mathrm{k}_{17}^{\prime}=0.06$, $\mathrm{Km}_{17}=15, \mathrm{~K}^{\prime} \mathrm{m}_{17}=15, \overline{\mathrm{y}}_{7}=300$

Table 1: Parameter values, molar concentrations are in $\mathrm{nM}$ and reaction rate constants are in $\mathrm{nM} / \mathrm{s}$. 